\title{
DosSIER
}

Territorio, políticas públicas y salud. Hacia la construcción de un enfoque multidimensional

Magdalena Chiara

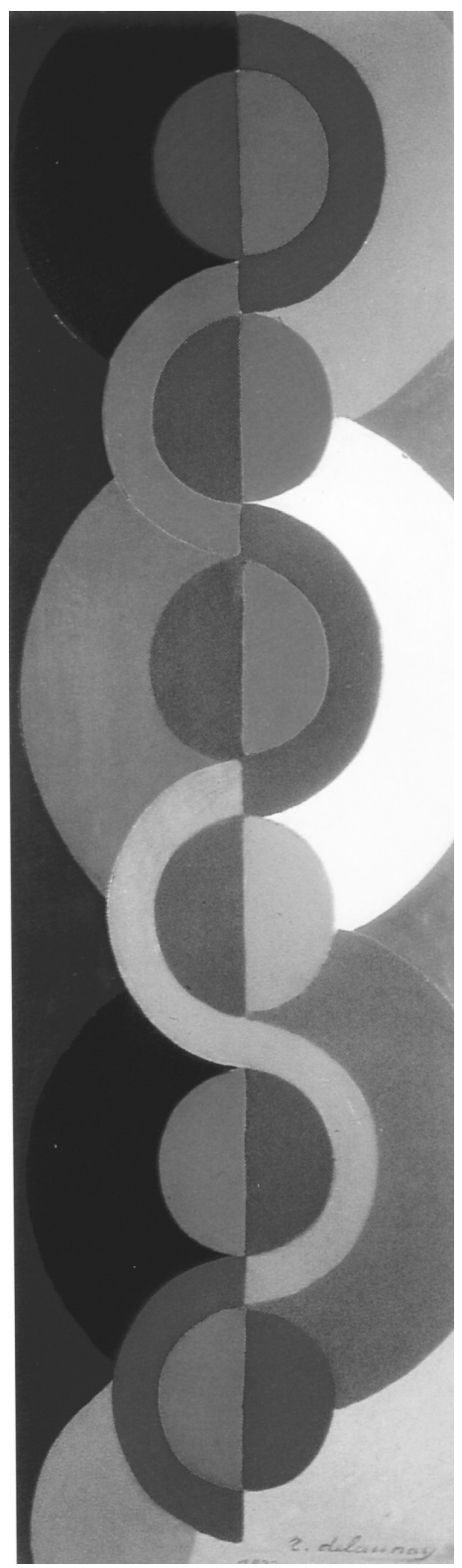




\section{Territorio, políticas públicas y salud. Hacia la construcción de un enfoque multidimensional para la investigación*}

\section{Territory, Public Policies and Health. Towards a Construction of a Multidimensional Approach for Research}

\section{Território, políticas públicas e saúde. Para a construção de um enfoque multidimensional para pesquisa}

Fecha de recepción: 28-08-15 Fecha de aceptación: 18-03-16 Disponible en línea: 03-05-16 doi:10.11144/Javeriana.rgyps15-30.tpps

Cómo citar este artículo:

Chiara M. Territorio, políticas públicas y salud. Hacia la construcción de un enfoque multidimensional para la investigación. Rev. Gerenc. Polít. Salud. 2016; 15(30): 10-22. http://dx.doi.org/10.11144/Javeriana. rgyps15-30.tpps 


\section{Resumen}

Objetivo: este trabajo propuso un marco conceptual para superar las limitaciones que enfrentan las aproximaciones disciplinares al analizar los procesos de implementación de las políticas de salud en el territorio. Método y marco de referencia: puso en diálogo contribuciones de tres campos: los estudios sobre desempeño en la atención de la salud, los aportes teóricos de la geografía y las investigaciones sobre políticas públicas e implementación. Resultados: en esta aproximación es posible captar fenómenos y procesos que, desde cada una de las miradas disciplinares particulares, resultan opacos al análisis: por una parte, concebir a la atención de la salud como un punto en una trama que compromete distintas nociones de territorio; por la otra, reconocer que en el momento de la implementación en el territorio tiene lugar un proceso de recreación de las instituciones en el que se ponen en juego y negocian distintas lógicas de regulación.

Palabras clave: políticas públicas; política de salud; estudios interdisciplinarios; geografía; atención primaria de salud; implementación de Plan de Salud.

\section{Abstract}

Objective: This paper proposed a conceptual framework to overcome the limitations the disciplinary approaches face when analysing the processes of implementation of health policies on the territory. Method and framework: With this purpose, it established links between the contributions from three fields: the studies on the performance on health care, the theoretical contributions provided by geography, and the research on public policies and implementation. Results: As a result of this approach, it is possible to capture phenomena and processes that, from each of the particular disciplinary perspectives, are opaque for the analysis: on one side, conceiving health attention as a point within a whole that compromises different notions of territory; on the other hand, recognise that at the moment of the implementation on the territory there is a process of recreation of the institutions, where they appear on scene and negotiate different regulation logics.

Keywords: public policies; health policy; interdisciplinary studies; geography; primary health care; Health Plan Implementation.

\section{Resumo}

Objetivo: este trabalho propôs um quadro conceptual para superar os constrangimentos que as aproximações disciplinares enfrentam para analisar os processos de implementação das políticas de saúde no território. Método e quadro de referência: coloco em diálogo contribuições de três campos: os estudos sobre desempenho no atendimento de saúde, as contribuições teóricas da geografia e as pesquisas sobre políticas públicas e implementação. Resultados: nesta aproximação é possível capturar fenômenos e processos que, desde cada uma das miradas disciplinares particulares, resultam obscuros para a análise: por um lado, conceber o atendimento de saúde como um ponto em uma trama que compromete diferentes noções de território; por outro, reconhecer que na hora da implementação no território tem lugar um processo de recriação das instituições em que entra em jogo e negocia diferentes lógicas de regulamentação.

Palavras-chave: políticas públicas; política de saúde; estudos interdisciplinares; geografia; atenção primaria em saúde; implementação de Plano de Saúde. 


\section{Introducción}

Las desiguales condiciones de vida, los procesos de movilidad, las barreras en el acceso y la relación entre los servicios, son algunos de los problemas que ponen en evidencia la relevancia de la dimensión territorial en las políticas de salud.

No obstante la contundencia de esas experiencias, la relación entre política sanitaria y territorio todavía es abordada desde campos disciplinares diferentes, sin llegar a conformar un corpus conceptual que permita comprender la complejidad de los procesos que desafían a la investigación en salud.

En ese contexto, este trabajo propone un enfoque multidimensional destinado a investigar las relaciones de mutuo condicionamiento que existen entre "territorio" y "política sanitaria", haciendo dialogar tres vertientes de aportes de tres campos diferentes: los estudios de salud pública, la geografía y el análisis de políticas públicas.

El texto está organizado en cuatro partes. Una primera parte pasa en limpio cómo ha aparecido el territorio en las orientaciones de la Organización Panamericana de la Salud y de la Organización Mundial de la Salud (ops/OMs), haciendo un contrapunto en una segunda parte con las cuestiones sobre las cuales echa luz la investigación. Tomando los desafíos que deja planteado el diálogo entre estas dos aproximaciones (institucional y académica), el tercer apartado pone el foco en la necesidad de conceptualizar esta relación, para lo cual incursiona en tres dominios del conocimiento: los estudios del desempeño de la atención de salud, los aportes conceptuales de la geografía y las contribuciones del análisis de políticas. Para finalizar, el último apartado pasa en limpio las contribuciones de esta perspectiva para el análisis.

\section{La emergencia de lo territorial en salud: el derrotero de los planteamientos de la ops/oms}

Una mirada desprevenida podría concluir que el territorio ha estado ausente en las orientaciones que animaron la política sanitaria; sin embargo, la reconstrucción de los planteamientos de la ops/OMs permite matizar este postulado. Como plantean Chiara y Ariovich (1), el recorrido de los últimos sesenta años pone en evidencia distintas maneras a través de las cuales el territorio fue emergiendo en las recomendaciones de estos organismos.

A mediados del siglo pasado, el combate de las enfermedades transmisibles organizó el modo de pensar y de hacer política sanitaria. La noción de "erradicación", fundada en adelantos científicos como el descubrimiento de vacunas y del DDT, animó la acción de la OMs y de los gobiernos, identificando a la viruela y a la malaria como enfermedades que debían ser erradicadas. En ese contexto, se forjó un modelo de intervención denominado "programas verticales", que sigue vigente y convive con otras formas (denominadas "horizontales") de concebir la política sanitaria.

Inscripto en una lógica top-down, y tal como plantean Tobar et al. (2), el modelo de los "programas verticales" buscó resolver en un tiempo acotado un problema de salud, a través de una estrategia que comprendía la fijación de normas de atención, la organización adecuada de los recursos y la racionalización del uso de la tecnología. Estas ideas fuerza se tradujeron en una concepción mecanicista de la organización sanitaria que jerarquizaba el poder central y buscaba un despliegue geográfico cercano a la idea de "control militar del territorio". 
El éxito del combate de la viruela validó esta forma de intervención, pero tuvo resultados diferentes en relación con la malaria, virando de la noción de "erradicación" a la de "control"; la enfermedad era considerada un problema de carácter local que afectaba a áreas particulares. Comenzaba a hacerse evidente la relevancia de los atributos singulares de cada territorio, difícilmente abordables desde estrategias homogéneas.

En contraste con esta lógica, se fue delineando otra forma de concebir la política sanitaria que tenía como ventaja una mayor sensibilidad para captar estas diferencias.

La "aproximación horizontal” dominó gran parte de las propuestas de la ops/oms a partir de finales de la década de los setenta. La Declaración de Alma Ata de 1978 definió y otorgó reconocimiento internacional al concepto de "atención primaria de la salud (APS)", estrategia fundada en la prevención de la enfermedad y en la promoción de la salud. El "territorio como el espacio de lo próximo y la participación” de la comunidad estuvo presente en aquellas definiciones.

Posteriormente, se sucedieron distintas definiciones de la APS: unas fueron sinónimo de primer nivel de atención, otras tuvieron un alcance selectivo y otras se integraron con los enfoques de los determinantes sociales y de las redes de servicios (3). Estas diferencias se forjaron en el contexto de los cambios que se sucedieron en el modo a través del cual pensar la política social y el rol del Estado; la APs fue adquiriendo así distintos contenidos, a la vez que mantenía ciertos rasgos comunes referidos a la noción de gestión de "lo próximo", a la jerarquización de la escala "barrial” y a la noción de "comunidad". La APS persistió en las distintas estrategias que se fueron sucediendo, mostrando capacidad para permear los discursos en las orientaciones de políticas y organizar la práctica de los equipos de salud, a la vez que la capacidad para ser actualizada a los nuevos escenarios.

$\mathrm{El}$ actor privilegiado por la APS fue y sigue siendo el equipo de salud, secundado por las organizaciones sociales comunitarias. La propuesta de los Sistemas Locales de Salud (Silos) amplió el espacio de proximidad del equipo de salud a una escala mayor, construida a través de los procesos de descentralización, en donde adquiere particular relevancia el gobierno local como actor político y administrativo.

En la Declaración de Atenas de 1998, la oms pasó en limpio una experiencia que había comenzado algunas décadas atrás, la propuesta de municipios y ciudades saludables; en América Latina, adoptó el nombre de Municipios, Ciudades y Comunidades Saludables (4). En esta propuesta, el espacio local cobra un doble significado: es la unidad territorial, a la vez que el conjunto poblacional de referencia para el diseño de acciones de promoción y prevención en salud.

Esta propuesta parece situarse en un lugar de "bisagra" con otra forma de asumir el territorio, más fecunda para el análisis pero menos potente en lo político-institucional: la estrategia de los "determinantes sociales de la salud". Aunque sus raíces se remontan al informe Lalonde (5), se cristaliza en el año 2008 en el documento elaborado por la Comisión de los Determinantes Sociales de la Salud. Esta perspectiva postula que la mala salud de los pobres, el gradiente social de salud y las grandes desigualdades sanitarias entre y dentro de los países son consecuencia de una distribución desigual del poder, de los ingresos, de los bienes y de los servicios. El informe postula que la distribución desigual de experiencias perjudiciales para la salud no es un fenómeno "natural", sino el resultado de la combinación de políticas y programas sociales deficientes, arreglos económicos injustos y una mala gestión política. El abordaje 
intersectorial que encierra este planteamiento amenaza con diluir la responsabilidad de las instituciones sanitarias. Tal como plantean Chiara y Ariovich (1), aunque la inequidad tiene al territorio como escenario, refiere a los individuos y a los grupos; en aquellos casos en que converge con la propuesta de Municipios, Ciudades y Comunidades Saludables, parece adquirir mayor densidad.

Más recientemente, el planteamiento de las Redes Integradas de Servicios de Salud (RISS), también propugnada por la ops/OMS, regresa a los servicios capitalizando contribuciones del enfoque de APs vinculadas a los estudios del desempeño en la atención. Respondiendo a los problemas derivados de la "fragmentación", la conformación de redes permitiría integrar los servicios de salud, aportando equidad y eficiencia a los usuarios.

En este recorrido se advierte una transformación del lugar del territorio en el sector salud, aunque muestra ciertas insuficiencias para comprender la conflictividad derivada de su historia y en cuyo contexto se constituye la territorialidad.

\section{Temas y problemas en la investigación}

En diálogo con esta construcción de la noción de territorio presente en los documentos institucionales, los resultados de las investigaciones ponen en evidencia procesos que hablan de otra complejidad.

En el plano de la "salud de la población", algunas investigaciones parecen confirmar el (o abonar al) enfoque de los determinantes sociales. Los estudios sobre desigualdades sociales en salud, como los de Barata (6) o los de Mackenbach et al. (7) muestran cómo inciden

14 los factores urbanos, ambientales y socioeconómicos en la situación de salud de la población.
La importancia que adquieren los efectos de localización y los modos como afectan las oportunidades de la población y la movilidad como factor de inequidad, han sido aspectos abordados de manera recurrente en las investigaciones que se ocupan de los "servicios". Siguiendo a Gutiérrez (8), se entiende por movilidad una práctica social de viaje en la que se ponen en juego deseos y necesidades de desplazamiento (o requerimientos de movilidad) y capacidades de satisfacerlos.

En este dominio se destaca el trabajo de Katzman (9), quien analiza cómo el contexto barrial afecta directa o indirectamente a las estructuras de oportunidades asociadas a la vida en la ciudad y dibuja diferentes trayectorias o "rutas al bienestar". Los estudios de movilidad realizados por Gutiérrez (8) muestran que los traslados y sus costos en tiempo y dinero son factores que intensifican las inequidades en la distribución de los servicios de salud en áreas metropolitanas; el estudio de Paganini et al. (10) muestra, por su parte, los usos diferenciales que hace la población de los servicios de salud. Estos estudios llaman la atención acerca de los modos como el deterioro de los servicios universales refuerza los efectos derivados tanto de la localización de los servicios como de las condiciones de vida de la población, en un proceso de "mutua determinación" que se construye en la interfase del primer nivel.

El ámbito de las "políticas" es quizás el que exige tomar mayor distancia de las conceptualizaciones presentes en las orientaciones de los organismos a las que se hizo referencia en el apartado anterior. Los procesos de toma de decisiones de la política sanitaria que tienen lugar en la conformación de la agenda, el diseño y su implementación, comprometen diferencias, tensiones y conflictos entre actores que se despliegan en el territorio. Los mayores aportes provienen de los estudios sobre las reformas en general, como los de Almeida (11), 
Fleury (12) y Ugalde sobre la descentralización en particular, la naturaleza de sus procesos y sus resultados (13). Una parte de las investigaciones sobre política sanitaria se orienta a los estudios de APS, como los trabajos realizados por Almeida et al. (14) y Stolkiner et al. (15).

Estas investigaciones devuelven un territorio complejo en sus determinaciones, atravesado por los problemas de la movilidad y estructurado a partir de la trama de actores de la implementación de las políticas.

\section{Hacia la construcción de un marco conceptual}

El recorrido realizado en los apartados anteriores muestra que el territorio sigue estando presente en las orientaciones de la ops/OMs, aunque con ciertas insuficiencias para dar cuenta de la complejidad de los problemas que ponen en evidencia los estudios territoriales en salud.

El marco de análisis que aquí se presenta parte de las contribuciones de las investigaciones sobre "desempeño en la atención de la salud" y las pone en diálogo con los modos de conceptualizar el territorio que aporta la "geografía", teniendo como mediación a los estudios de "políticas públicas". Interesa en este apartado proponer un conjunto de conceptos desde los cuales dar cuenta de los procesos de toma de decisiones que tienen lugar en el territorio (tanto en el diseño como en la implementación de las políticas), prestando atención a la singularidad que enfrenta el sector salud, y desde estas particularidades establecer "puentes" con los otros dominios.

\section{Acerca de la complejidad de la atención de la salud}

Para analizar los servicios de salud, Belmartino (16) distingue tres ejes en torno a los cuales se estructuran los problemas de los sistemas de salud: la "relación entre población y proveedores", la "relación entre financiadores y proveedores" y, por último, la "interacción entre las organizaciones de financiamiento y/o provisión de atención médica y las autoridades locales y regionales". Dados los propósitos del trabajo, interesa poner el foco en la primera retomando las dimensiones que han organizado parte de los estudios sobre desempeño de los servicios desde la estrategia de la atención de la salud que identificaron Starfield et al. (17).

En este análisis se rescatan seis atributos de los "estudios sobre el desempeño" en tanto "dimensiones de análisis de la atención", utilizados en un estudio local por Ariovich y Jiménez (18):

- "Accesibilidad" o "puerta de entrada": alcanza a aquellos factores institucionales que inciden en el acceso de la población a los servicios de salud, incluidos los procedimientos administrativos para ser atendidos.

- "Continuidad": refiere a la existencia de un equipo de salud con el que el paciente se relacione habitualmente $\mathrm{y}$ pueda construir un vínculo.

- "Longitudinalidad": alude al alcance de las prestaciones, tanto preventivas, de diagnóstico, de tratamiento como de rehabilitación; supone una más oportuna y apropiada identificación de los problemas.

- “Integralidad": comprende la organización de respuestas sanitarias acordes a las necesidades del paciente, la familia y la comunidad.

- "Capacidad resolutiva": determina la proporción de problemas de salud que pue- 
den ser abordados a través del equipo de salud, o bien por medio de derivaciones.

- "Coordinación asistencial": refiere a la capacidad que alcanzan los servicios de salud para procurar (de manera concurrente) un objetivo asistencial en común al paciente, sin que se produzcan desajustes que terminen perjudicándolo, todo esto de manera independiente de cuál sea el lugar en el que el paciente sea atendido.

Se trata de atributos pensados originalmente para normalizar un modelo (la APS), que se adoptan en esta propuesta como "dimensiones pertinentes para describiry analizar" los procesos de atención y sus relaciones con el territorio.

Siguiendo a Belmartino (16), es posible identificar nueve funciones propias de la atención, con independencia de quién las toma a su cargo y en qué nivel del sistema se cubren. Estas funciones serían: (a) ser primer punto de contacto del paciente con la atención profesional; (b) hacerse cargo de clarificar la demanda; (c) brindar información, seguridad y consejo; (d) realizar el procedimiento diagnóstico; (e) aplicar tratamiento; (f) involucrar a otras disciplinas; (g) ocuparse de la coordinación cuando están involucrados otros profesionales, de manera de asegurar continuidad; (h) garantizar la prevención resultante del conocimiento del paciente y sus condiciones de vida; e (i) registrar la información en historias clínicas para garantizar la coordinación y la continuidad de la atención prestada.

Como se puede advertir, casi la totalidad de los atributos de la atención antes referidos ("accesibilidad" o "puerta de entrada", "continuidad", "longitudinalidad", "integralidad", "capacidad resolutiva" y "coordinación asistencial") se manifiestan en estas

16 funciones, al margen de "quiénes" las ejerzan o qué "nivel de atención" las tome a cargo.
La literatura sobre atención de salud insiste en dos cuestiones de particular relevancia para el análisis de las tensiones que se suceden en el territorio; la primera remite a la "noción de trabajo en red" y la segunda al "rol del médico generalista".

La propuesta de Redes Integradas de Sistemas y Servicios de Salud es la expresión más orgánica de la primera cuestión. La formación de redes es una de las estrategias que busca hacer efectiva una parte importante de los atributos que caracterizan la atención de la salud como modelo; la "longitudinalidad", la "capacidad resolutiva" y la "coordinación asistencial" son atributos de la atención que requieren la formación de redes en el territorio.

Con relación a la segunda cuestión, el médico generalista (y los distintos actores que forman parte del "equipo de salud") asume un rol articulador de las funciones antes aludidas, pudiendo estar involucrado tanto en relaciones "hacia arriba" con la atención hospitalaria, "lateralmente" con servicios de emergencias, enfermería o servicios sociales, o bien "hacia abajo" con servicios escolares u ocupacionales.

Más allá del énfasis puesto en la figura del profesional médico (que aparece como protagonista único de estos múltiples roles), la "nodalidad" es un atributo de la atención que no parece estar determinado exclusivamente por cuestiones vinculadas al campo de las ciencias médicas, sino por el espacio donde se desarrolla.

\section{Del "espacio" al "territorio": aportes de la geografía}

La segunda entrada del marco de análisis que aquí se propone remite a los estudios territoriales, en particular a las contribuciones de la geografía. 
Los modos de conceptualizar la noción de territorio en este campo han ido abandonando progresivamente visiones espacialistas, moviéndose hacia concepciones que dan cuenta de su carácter histórico. Sin embargo, la sola invitación a desnaturalizar el carácter dado del territorio, no parece ser suficiente para hacerlo operativo como dimensión (territorial) en el análisis de la política sanitaria.

Desde una perspectiva que destaca el carácter histórico, dinámico, determinante y determinado por la acción de los actores, Dematteis y Governa (19) definen "la territorialidad [...] como [una] relación dinámica entre los componentes sociales (economía, cultura, instituciones, poderes) y aquello que de material e inmaterial es propio del territorio donde se habita, se vive, se produce". Esta perspectiva enfatiza los procesos enlazados que se dan entre los actores, la estructura social y el territorio en el seno de los cuales se inscribe el campo de la política sanitaria.

Así concebido lo territorial, interesa rescatar de estos aportes dos líneas de análisis: el modo como concebir lo local y la perspectiva relacional del territorio.

Con relación a la primera línea de análisis, se propone retomar aquellos planteamientos que ven a "lo local como heterogeneidad juxtapuesta”, expresión de distintas escalas e intereses, "micromundos" atrapados en el espacio, cuyos conflictos deben ser gestionados. Amin (20) discute con la perspectiva convencional sobre las regiones que define a lo local en oposición a lo global, confiriendo a estos polos atributos que alcanzan el carácter de (en palabras del autor) "caricaturas":

\section{[...] lo local [es] [...] percibido como el} espacio de lo íntimo, de lo familiar, lo cercano, lo encarnado; es decir, como un espacio esencialmente separado y distinto del espacio global que se concibe como lo lejano, lo abstracto, lo virtual, lo invasivo, lo hegemónico. [En esa discusión sostiene que] [...] las configuraciones y los límites espaciales no son ya necesariamente territoriales o escalares, puesto que el interior y el exterior social, económico, político y cultural se constituyen a través de topologías de redes de actores que están siendo cada vez más dinámicas y diversas en la conformación espacial). (20: 77-8)

Esta perspectiva conduce a cuestionar una visión de lo local basada en la idea de "ámbitos territoriales comunes o de una cultura territorial cohesionada", construyendo, en palabras del autor, "caricaturas que se presentan con un cierto grado de veracidad acerca de la esfera pública” (20: 85).

Interesa retomar sus aportes para mirar lo local, el ámbito de la descentralización y el territorio de referencia más próximo de la política sanitaria, como "heterogeneidad yuxtapuesta dentro de una estrecha proximidad espacial, y como lugares de múltiples geografías de afiliación, vínculo y flujo" (20: 86). Desde esta perspectiva, el ámbito de la política local puede ser considerado un campo de reivindicaciones, acuerdos y coaliciones (frágiles y temporales) que son el resultado de dinámicas cambiantes e interconectadas.

Desde estas aproximaciones, el propio concepto de "comunidad" — tan caro al enfoque de la APS- se pone en cuestión. En ese sentido, Dematteis y Governa destacan: "la dificultad de hablar de comunidad y, al mismo tiempo, la urgencia de los problemas que $[\ldots]$ se ocultan dentro de esta palabra y que se difuminan en las 'trazas' de la confianza, la reciprocidad y la identidad" (19: 36-37). Aquella perspectiva comunitarista de lo local conduce a una visión naturalizada del territorio, a lo que los mismos autores denominan un "territorio sin actores". 
A diferencia de las formulaciones más clásicas sobre el modo como se ha develado el territorio en el planteamiento de la APS, estos aportes cuestionan la visión ingenua de lo local como "lo íntimo" o "lo familiar" y desafían a desentrañar las complejidades comprendidas en la delimitación de una determinada área programática.

La segunda línea de análisis que se busca recuperar en esta aproximación refiere a la "perspectiva relacional del territorio", aproximación que propone interpretar los fenómenos sociales y las demandas realizando una distinción entre "territorios de proximidad" y "territorios de conectividad".

En el análisis de los procesos de configuración del territorio, Catenazzi y Da Representacao (21) distinguen entre un área contigua (asociada a la métrica topográfica) y una red (a la métrica topológica), lo que se corresponde — en el análisis de los actorescon lógicas de apropiación y acumulación diferentes:

\begin{abstract}
Para quienes defienden una relación muy fuerte entre territorio y apropiación, el territorio es una entidad única, estrictamente definida y delimitada por el control ejercido sobre el espacio. Por el contrario, si la configuración espacial adoptada depende de los recursos movilizados y de diferentes modalidades de control (material y simbólico), el territorio puede ser tanto un área contigua, como archipiélagos o reticular. (21: 122)
\end{abstract}

De manera convergente con estas autoras, Dematteis y Governa distinguen entre la territorialidad que se expresa en una estrategia inclusiva que regresa a lo local y al control, y un segundo sentido en el que la territorialidad remite al conjunto de relaciones que una sociedad mantiene con el exterior y con los demás agentes, con la ayuda de mediadores (19: 44).
En la misma línea, la idea de "territorio de conectividad" da cuenta no solo de la imprecisión de las fronteras, sino también de la noción de espacio "fractal" con que Amin caracteriza a lo local, de la multiplicidad de conexiones, significados e influencias que tienen lugar en el territorio, de modo que el "dentro y fuera" ya no están definidos localmente. Retomando palabras de este autor, en la arena local "los diferentes micro-mundos se encuentran atrapados en el mismo terreno y las presiones y los diferentes intereses deben ser gestionados y negociados activamente porque no existe ningún otro terreno" (20: 87).

En el campo de salud esta observación adquiere aún mayor relevancia, en tanto el "adentro" y el "afuera" pueden librarse en simultáneo en la atención médica, la cual aun estando localizada en una barriada de la periferia, requiere interconsulta a especialistas, estudios diagnósticos especializados y una relación con las comunidades científicas internacionales.

A manera de recapitulación, los estudios territoriales están llamados a desempeñar un papel capital en la desnaturalización del territorio. La noción de lo local como "heterogeneidad yuxtapuesta", que articula escalas y representa distintos micromundos atrapados en un espacio, permite regresar al "territorio de proximidad", dando cuenta de su complejidad y, a su vez, de la conflictividad que luego (en la implementación) enfrentan los equipos de salud.

\section{El papel mediador del análisis de políticas públicas}

El campo de las políticas públicas ofrece la posibilidad de construir "puentes" entre los estudios sobre atención de salud y la geografía. 
Los procesos a partir de los cuales se construye esta mediación permiten identificar, al menos, aunque no exhaustivamente, las siguientes cuestiones en torno a las cuales se organizan los aportes: el carácter construido del territorio en salud; las interfaces que se conjugan en las relaciones intergubernamentales; las tensiones y la fragmentación que producen la introducción de mecanismos de compra de servicios; y por último, las tensiones con la lógica sectorial.

La primera cuestión advierte acerca de que no existe un territorio predefinido para la política pública, sino que este es objeto de apropiación o construcción por parte de sus agentes.

En el campo de la política sanitaria, esta perspectiva invita a mirar no solo cómo los fenómenos estructurales ponen en evidencia la centralidad de la dimensión territorial, sino también su inversa, es decir, la capacidad que tiene la política sanitaria de "construir territorialidad". En este sentido, la instalación de infraestructura sanitaria produce "territorialidad", en la medida en que incorpora mayor dotación de servicios para una determinada población, a la vez que moviliza el desarrollo de otras actividades (transporte, servicios, nuevas infraestructuras).

Sin desconocer el impacto material de estas inversiones, cabe destacar la relevancia que tienen los "intangibles" de la política sanitaria, que van más allá de los recursos físicos o humanos. Cuestiones vinculadas al acceso (como la condición de gratuidad o los procesos de mercantilización de servicios, por ejemplo) o los mecanismos de coordinación, definen usos, condicionan los procesos de movilidad de la población y delimitan fronteras. Desde la acción de las políticas públicas, configuran — de distinto modo- un "afuera" y un "adentro" del territorio.
En este mismo sentido, el balance retrospectivo acerca de los procesos de descentralización en América Latina que hacen Jordana (22) y Gomá (23) demuestra que la transferencia de funciones hacia niveles subnacionales dio lugar a servicios muy desiguales. Esos procesos dibujaron un mapa muy diverso en las condiciones de acceso, en la utilización de los servicios y en los mecanismos de coordinación que los vinculan, diferencias que expresan distintos "modelos de atención" a través de los cuales los ciudadanos ejercen el derecho a la salud.

La segunda cuestión en torno a la cual se articulan los aportes de los estudios de políticas refiere a las interfases que se conjugan en las relaciones intergubernamentales. Se trata de un espacio complejo que está definido por el marco institucional, a la vez que por la dinámica de las relaciones entre los actores en la puesta en marcha e implementación de las políticas, tal como muestran los estudios comparados de Banting y Corbett (24).

Una tercera línea de investigaciones se vertebra en torno a los procesos implicados en el diseño e implementación de modelos de aseguramiento y los mecanismos de compraventa de servicios de salud, en los que se destaca el papel que desempeñaron las reformas institucionales, la dinámica con los actores del mercado y los impactos diferenciales en la fragmentación; un ejemplo de esta aproximación es el balance que hacen Laurell y Herrera Ronquillo (25) sobre las experiencias de Chile, Colombia y México.

La dinámica que establecen los actores en el devenir de las políticas pone en evidencia decisiones y sistemas de justificación que movilizan distintos conceptos de territorio, pudiendo establecerse diferencias - no lineales-entre niveles de gobierno. Por una parte, la existencia de una lógica de apropiación sobre un "área contigua", a la que hacían 
referencia Catenazzi y Da Representacao (21) (asociada a una métrica topográfica), especialmente protagonizada por los actores locales, que lleva implícito el interés por desarrollar los cuatro niveles de complejidad en la atención bajo una misma jurisdicción. Por la otra, la concepción de "territorios en red" (asociada a una métrica topográfica) en tanto sistemas abiertos pero que requieren la construcción de articulaciones intergubernamentales que suponen procesos complejos de coordinación en sus aspectos organizacionales, operativos y de financiamiento.

Por último, una cuestión de suma importancia que es preciso indagar en la relación entre política sanitaria y territorio refiere a la singularidad de lo "sectorial" y a sus diferencias con otras lógicas como la "territorial".

Siguiendo a Jolly (26), la "lógica sectorial" está caracterizada por tres atributos que la distinguen de otras que organizan a las políticas públicas: está regida por el principio de especialización; su dinámica intra e inter organizacional está dominada por la legitimidad que le confiere la legalidad de las normas; $y$, por último, la importancia que adquiere el conocimiento científico-técnico en sus sistemas de justificación. Estos atributos, que se expresan con fuerza en salud (pero que están presentes en otros campos de la política social), están reforzados por otro que lo caracteriza como sector: el hecho de que la "imagen de los profesionales médicos" sea el principal marco de referencia para la acción de las políticas.

La "lógica territorial", por otra parte, se sostiene en la horizontalidad de las relaciones entre los actores, en la particularidad de sus intereses y perspectivas, poniendo así "blanco sobre negro" la integralidad de sus demandas.

Ambas lógicas, "sectorial" y "territorial” tensionan en la implementación de las políticas, no ya por problemas o imprevisiones de su diseño, sino por lo naturaleza misma de los procesos que tienen lugar con la territorialización de las políticas.

Asumir esta tensión en el análisis de la política sanitaria supone dar cuenta de los modos a través de los cuales se combina un doble rol de la acción de gobierno: el de "agente regidor" impuesto por las normas y dominado por aquella lógica sectorial, y el "actor gobernante" impulsado por la complejidad de las demandas sociales y políticas que se expresan en el territorio.

La convivencia en tensión de estas dos lógicas en los procesos de implementación de las políticas de salud, la "sectorial" (caracterizada por la verticalidad, la normatividad y la parcialidad de la especialización) y la "territorial” (horizontal, política, holística, preocupada por la cohesión), interpelan al analista a dar cuenta de las "formas híbridas de regulación" en las que "gobierno" y "gobernanza" se combinan en el territorio.

\section{Conclusiones: nuevos abordajes para la investigación en políticas de salud}

El acercamiento que aquí se propone pone la mirada sobre los estudios acerca de la atención de salud y, tomando distancia de una aproximación normativa, busca construir un enfoque desde el cual analizar y comprender lo que sucede en el territorio con las políticas de salud.

Los aportes que este trabajo pone en diálogo revelan - al menos - dos nudos que considerar en el análisis y el diseño de las políticas de salud en el territorio. 
En primer lugar, que la atención de la salud se constituye en un "punto" en una trama de relaciones que compromete en simultáneo dos nociones de territorio, "de proximidad" y "de conectividad". Esta aproximación permite interpretar las dimensiones del desempeño (accesibilidad, continuidad, longitudinalidad, integralidad, coordinación) en función de estos diferentes conceptos de territorio; lejos de ser un fenómeno aislado, la práctica de la atención parece relacionarse - en primera instancia - con la noción de "territorio de proximidad" al centro de salud, "puerta del acceso" al sistema, espacio de realización de la "integralidad" y la "longitudinalidad".

No obstante la fuerte presencia del "territorio de proximidad" en la atención primaria de la salud, otra acepción de este concepto se ve interpelada —en simultáneo- en la práctica del equipo de salud, en la medida en que la atención médica requiere una relación fluida con otros niveles (para la realización de diagnósticos especializados, derivaciones a especialistas o llevar a cabo prácticas de mayor complejidad). Así, la "coordinación asistencial" (otra dimensión del desempeño de la atención) interpela al "territorio de la conectividad" o "territorio de la red". De este modo, en la práctica de los equipos de salud se movilizan distintas nociones de territorio.

Esta aproximación multiescalar de la atención reenvía a un segundo nudo que anida en problemas clásicos de los estudios de políticas; en el momento de la implementación en el territorio tiene lugar un proceso de recreación de las instituciones en el nivel local, lo que Bifulco (27) denomina "cuasi instituciones". Este concepto reconoce que la legitimidad en la implementación no está depositada exclusivamente en las instituciones organizadas bajo la "lógica sectorial" (ministerio de salud, secretaría de salud, hospital, director del centro de salud), sino que en esos procesos intervienen interacciones de naturaleza contractual con actores políticos (jefes comunales, concejales, secretarios, legisladores, gobernadores) y de mercado (aseguradoras, unidades compradoras de servicios, prestadores) que forman un entramado bastante más complejo. Así, en la implementación se ponen en juego dos lógicas de regulación diferentes: la "sectorial" (vertical, normativa y regida por la legitimidad que le confiere la evidencia científica) y la lógica de regulación "territorial" (holística e impregnada por las miradas y los intereses de los actores).

Los nudos que ilumina esta perspectiva dan cuenta de la existencia de pliegues, fisuras y tensiones que no suelen estar visibles en los enfoques más clásicos de política sanitaria, pero cuya comprensión parece ser clave en la investigación para comprender su dinámica y, en la gestión, para transitar procesos de cambio.

\section{Referencias bibliográficas}

1. Chiara M, Ariovich A. Luces y sombras sobre el territorio. Reflexiones en torno a los planteamientos de la ops/oms en América Latina. Cad Metropole [Internet]. 2013; 15 (29): 99-122. Disponible en: http://www.cadernosmetropole.net/download/ cm_artigos/cm29 253.pdf.

2. Tobar F, Gürtler $\overline{\mathrm{R}}$, Yadón Z, Medici A. Introducción. En: Descentralización y gestión del control de las enfermedades transmisibles en América Latina [Internet]. Buenos Aires: ops; 2006. Disponible en: http://cidbimena.desastres.hn/filemgmt/files/ res-descentralizacion.pdf.

3. Organización Panamericana de la Salud (ops/OMs) La renovación de la atención primaria de salud en las Américas. Documento de posición de la ops/ OMS. 2005. Washington: ops/oms D.C. [Internet]. Disponible en: http://cursos.campusvirtualsp.org/ pluginfile.php/14021/mod_resource/content/0/CPS/ modulo1/m1lecturasprincipales/Renovacion_de la_APS_1-16_.pdf.

4. Organización Panamericana de la Salud (ops/OMs). Municipios y ciudades saludables: recomendaciones para la evaluación dirigidas a los responsables de las políticas en las Américas. Washington, D.C.: oPs; 2005.

5. Lalonde M. A new perspective of the health of $\mathrm{Ca}$ nadians. A working document. Minister of Supply and Services Canada. 1981

6. Barata RB. Epidemiologia social. Rev Bras Epidemiologia [Internet]. 2005; 8 (1): 7-17. Disponible 
en: http://www.scielo.br/scielo.php?script=sci arttext\&pid=S1415-790X2005000100002.

7. Mackenbach J, Bakker M, Judge, K. Tackling socioeconomic inequalities in health; an analysis of European experiences. Lancet. 2000; 362 (9393): 1409-14.

8. Gutiérrez A. La movilidad de la metrópolis desigual: el viaje a la salud pública y gratuita en la periferia de Buenos Aires. En: XII Encuentro de geógrafos de América Latina, 2009, Montevideo [Internet]. Disponible en: http://observatoriogeograficoamericalatina.org.mx/egal12/Geografiasocioeconomica/Geografiaurbana/85.pdf.

9. Katzman R. Marco conceptual sobre activos, vulnerabilidad y estructura de oportunidades. Montevideo: Eclac; 1999 [Internet]. Disponible en: http://www.cepal.org/publicaciones/xml/6/10816/ LC-R176.pdf.

10. Paganini JM, Rossen M. Los caminos de la salud en el área metropolitana de Buenos Aires. Políticas Públicas y Derechos Humanos 11. Defensoría del Pueblo de la CABA: 2010 [Internet]. Disponible en: http://www.saludyderechos.org.ar/2012/09/loscaminos-de-la-salud-en-el-area 5.html.

11. Almeida, C. Equidade e reforma setorial na América Latina: um debate necessário. Cad. Saúde Pública [Internet]. 2002; 18 (Supl): 23-36. Disponible en: http://www.scielo.br/pdf/csp/v18s0/13790.pdf.

12. Fleury S. Salud y democracia en Brasil. Valor público y capital institucional en el Sistema Único de Salud. Rev Salud Colectiva [Internet]. 2007; 3(2): 147-57. Disponible en: http://www.unla.edu.ar/ saludcolectiva/revista08/4.Salud $\% 20 y \% 20$ Democracia\%20en\%20Brasil.pdf.

13. Ugalde A, Homedes N. La descentralización de los servicios de salud: de la teoría a la práctica. Rev Salud Colectiva [Internet]. 2008; 4 (1): 31-56. Disponible en: http://www.unla.edu.ar/saludcolectiva/ revista10/3.La\%20descentralizacion $\% 20 \mathrm{de} \% 20$ los $\% 20$ servicios $\% 20$ de $\% 20$ salud.pdf.

14. Almeida C, Macinko J. Validação de metodologia de avaliação rápida das características organizacionais e do desempenho dos serviços de atenção básica do Sistema Único de Saude (sus) em nível local. Série Técnica Desenvolvimento de Sistemas e Serviços de Saúde, 2006.

15. Stolkiner A, Comes Y, Garbus P. Alcances y potencialidades de la Atención Primaria de la Salud en Argentina. Rev C S Col [Internet]. 2011; 16 (6): 2807-16. Disponible en: http://www.scielosp.org/ pdf/csc/v16n6/19.pdf.

16. Belmartino S. La gestión local de atención médica abordada como objeto de investigación. En: Chiara M, Di Virgilio MM y Miraglia M, organizadoras. Gestión local en salud: conceptos y experiencias. Los Polvorines: UNGs; 2008 [Internet]. Disponible en: http://www.ungs.edu.ar/cm/uploaded_files/ publicaciones/123_LU25.pdf.
17. Starfield B, Shi L, Macinko J. Contribution of primary care to health system and health. Rev The Milbank Quartery. [Internet] 2005; 83 (3): 457-502. Disponible en: http://www.commonwealthfund.org/ usr_doc/Starfield_Milbank.pdf.

18. Ariovich A, Jimenez C. Accesibilidad a la atención de la salud en el municipio de Malvinas Argentinas. Los Polvorines: Ungs; 2014 [Internet]. Disponible en: http://www.ungs. edu.ar/areas/publicaciones/643/accesibilidada-la-atencion-de-la-salud-en-el-municipio-demalvinas-argentinas.html.

19. Dematteis G, Governa F. Territorio y territorialidad en el desarrollo local. La contribución del Modelo SLOT. Boletín de la AGE 2005; 39: 31-58. Disponible en: http://dialnet.unirioja.es/servlet/ articulo? codigo $=1161234$.

20. Amin A. Regiones sin fronteras: hacia una nueva política del lugar. Ekonomiaz [Internet]. 2005; 58 (1 ${ }^{\text {er }}$ cuatrimestre). Disponible en: file:///C:/Users/ Ultrabook/Downloads/Dialnet-RegionesSinFronteras-2008985.pdf.

21. Catenazzi A, Da Representacao N. Acerca de la gestión de la proximidad. En: Chiara My Di Virgilio MM, compiladoras. Gestión de la política social. Conceptos y herramientas. Buenos Aires: UNGS/ Prometeo; 2009.

22. Jordana J. Relaciones intergubernamentales y descentralización en América Latina: una perspectiva institucional. Serie Documentos de Trabajo I-22UE. Indes/ BID. 2001 [Internet]. http:// publications.iadb.org/handle/11319/1252?localeattribute $=$ es.

23. Gomá R, Jordana J. Descentralización y políticas sociales en América Latina. Barcelona: Cidob; 2004.

24. Banting K, Corbett S. Federalism and health care policy: an introduction. En: Health Policy and Federalism A comparative perspective. Kingston: Queen's University Institute of Intergovernmental Relations; 2002.

25. Laurell A, Herrera Ronquillo J. La segunda reforma de salud. Aseguramiento y compraventa de servicios. Salud Colectiva, Buenos Aires [Internet]. 2010 mayo - agosto; 6 (2): 137-48. Disponible en: http://www.redalyc.org/articulo. oa? id=73115020002.

26. Jolly JF. Gobierno y gobernancia de los territorios, sectorialidad y territorialidad de las políticas públicas. Desafíos. 2005; 12: 52-85. Disponible en: http:/ www.scielo.org.co/pdf/papel/v12n2/v12n2a05.pdf.

27. Bifulco L. Governance e territorialização: o welfare local na Itália entre fragmentação e inovação. Cad Metropole [Internet]. 2012; 14 (27): 41-57. Disponible en: http://www.cadernosmetropole.net/ download/cm_artigos/cm27_227.pdf. 